\title{
A-769662, a direct AMPK activator, attenuates lipopolysaccharide-induced acute heart and lung inflammation in rats
}

\author{
MARYAM RAMESHRAD ${ }^{1,2}$, HAMID SORAYA ${ }^{3}$, NASRIN MALEKI-DIZAJI $^{1}$, \\ HALEH VAEZ $^{1}$ and ALIREZA GARJANI ${ }^{4}$
}

\author{
${ }^{1}$ Department of Pharmacology and Toxicology; ${ }^{2}$ Student Research Committee, Faculty of Pharmacy, \\ Tabriz University of Medical Sciences, Tabriz, East Azerbaijan 51656-65811; ${ }^{3}$ Department of Pharmacology, \\ Faculty of Pharmacy, Urmia University of Medical Sciences, Urmia, West Azerbaijan 54147-83734; \\ ${ }^{4}$ Cardiovascular Research Centre, Tabriz University of Medical Sciences, Tabriz, East Azerbaijan 51656-65811, Iran
}

Received April 28, 2015; Accepted December 2, 2015

DOI: $10.3892 / \mathrm{mmr} .2016 .4821$

\begin{abstract}
Activation of AMP-activated protein kinase(AMPK) has been indicated to produce an anti-inflammatory effect through the suppression of toll-like receptor (TLR) activity. In the present study, the investigation was designed to identify the effect of A-769662, a direct activator of AMPK on lipopolysaccharide (LPS)-induced acute lung and heart inflammation in rats. To induce inflammation, an intraperitoneal injection of LPS $(0.5 \mathrm{mg} / \mathrm{kg})$ was administered to Wistar rats. The inflammatory parameters and AMPK phosphorylation were then measured $9 \mathrm{~h}$ later. For the treatment group, A-769662 $(10 \mathrm{mg} / \mathrm{kg}$ ) was administrated intraperitoneally immediately prior to LPS injection. The results demonstrated that A-769662 attenuated the LPS-induced acute inflammation in the heart and lung tissue, as indicated by the significant reduction in myeloperoxidase activity $(\mathrm{P}<0.001)$ and inhibition of tissue damage. This was associated with a significant reduction in tumor necrosis factor- $\alpha$ serum levels $(\mathrm{P}<0.01)$ and peripheral neutrophils $(\mathrm{P}<0.001)$. Furthermore, A-769662 enhanced AMPK phosphorylation and downregulated the expression of MyD88, a TLR adaptor protein, in the heart tissue. Despite the anti-inflammatory effect of A-769662 on LPS-induced inflammation in the lung tissue, the drug produced no effect on the MyD88 expression levels or AMPK phosphorylation in the tissue. The results of the present study suggested that the administration of A-769662 results in an anti-inflammatory effect in the LPS-induced model of inflammation in rats. The
\end{abstract}

Correspondence to: Professor Alireza Garjani, Cardiovascular Research Centre, Tabriz University of Medical Sciences, Danesgah Street, Tabriz, East Azerbaijan 51656-65811, Iran

E-mail: garjania2002@yahoo.com; garjania@tbzmed.ac.ir

Key words: A-769662, lipopolysaccharide, AMP-activated protein kinase, toll-like receptor, inflammation anti-inflammatory activity was demonstrated in the heart and lung tissues and the effect on the cardiac tissue was indicated to be a result of AMPK activation, involving the suppression of TLRs.

\section{Introduction}

Sepsis is a systemic inflammatory response syndrome caused by severe infection, which is characterized by inflammation occurring in tissues that are remote from the infection. The inflammatory responses in sepsis are primarily initiated by the bacterial lipopolysaccharide (LPS), known as an endotoxin. During endotoxemia, LPS acts as a ligand for pattern recognition receptors known as toll-like receptors (TLRs), specifically TLR4 $(1,2)$. The binding of LPS to TLR4 activates either myeloid differentiation primary response 88 (MyD88) or Toll/IL-1 receptor domain-containing adaptor inducing interferon- $\beta$ (TRIF) downstream pathways $(2,3)$. MyD88 is an adaptor protein that is critical for various TLR activities. Stimulation of MyD88 increases the systemic and tissue levels of pro-inflammatory cytokines, including tumor necrosis factor- $\alpha$ (TNF- $\alpha$ ) and interleukin-6 (IL-6) through the translocation of nuclear factor (NF)- $\mathrm{B}$ to the nucleus. The increase in inflammatory cytokines and mediators following LPS exposure contributes to generalized inflammation (4,5). A severe immune response may lead to septic shock (6) along with a reduction in cardiac output and multiple organ injury (7), including lung and liver failure (8). The rate of mortality due to sepsis among intensive care unit patients is $30-50 \%$ (6).

Previous studies have identified that the activation of AMP-activated protein kinase (AMPK) led to suppressed expression levels and activation of TLR4 in heart tissues, in conditions associated with inflammation, such as myocardial infarction $(9,10)$. Numerous studies have demonstrated that AMPK activation prevents the inflammatory reaction, and a reduction in AMPK activity has been associated with increased inflammation (11-13). However, the association of AMPK activity and TLRs in inflammation, particularly in vital tissues, including lung and heart, remains unknown. 
AMPK is a serine-threonine protein kinase that has a critical role in cellular metabolism and function (14). It acts as a sensor of energy in cells and is activated when the nutrient supply or ATP is limited, or upon an increase in the demand of cellular energy. Therefore, metabolic inhibitors, hypoxia, myocardial ischemia, hypoglycemia, exercise, heat shock, osmotic stress, peroxynitrite and oxidative stress are notable AMPK activators (15). Following AMPK activation, energy-consuming processes, such as protein and glycogen synthesis are suppressed, and ATP generating pathways such as glucose uptake, glycolysis and fatty acid oxidation are activated (16).

At the molecular level, AMPK is a heterotrimer complex comprised of $\alpha, \beta$ and $\gamma$ subunits (17). Mammalian AMPK is sensitive to the AMP:ATP ratio and an increase in the ratio activates the enzyme. AMP binds to the $\gamma$ subunit of AMPK and induces a conformational change in the structure, that allosterically activates the $\alpha$ catalytic subunit, enhances phosphorylation of the Thr172 residue in the $\alpha$ subunit by upstream AMPK kinases, and inhibits the action of protein phosphatase 2C to dephosphorylate Thr172 $(18,19)$. A-769662 is a small non-nucleoside thienopyridine molecule with high specificity for AMPK. It directly binds to the $\beta$ subunit of AMPK to activate it (20) independently of the AMP:ATP ratio $(21,22)$. Furthermore, A-769662 activates the eukaryotic elongation factor kinase subsequent to AMPK activation and inhibits the energy-requiring protein synthesis, thus promoting ATP preservation during ischemia (20). In chondrocytes, A-769662 suppresses the matrix degradation response to inflammatory cytokines and the biochemical injury in which peroxisome proliferator-activated receptor- $\gamma$ coactivator $1-\alpha$ (PGC-1 $\alpha$ ) and forkhead box O3a mediate chondroprotection by A-769662-induced AMPK activation (23). A previous study suggested that preserving the AMPK activity by A-769662 in injured chondrocytes protects the cartilage matrix integrity and inhibits caspase-3 activation and catabolic response (24). A-769662 is a novel agent and compared with other activators of AMPK, including metformin and AICAR, few studies have investigated its anti-inflammatory effect. Therefore, for the present study, the effect of A-769662 on LPS-induced inflammation and tissue injury was investigated.

\section{Materials and methods}

Animals. Male Wistar rats $(240 \pm 10$ g, 8-weeks old) were purchased from Pasteur Institute of Iran (Tehran, Iran). A total of 15 rats were used (5 animals in each group). Animals were administered food and water ad libitum and were housed in the animal house of Tabriz University of Medical Sciences (Tabriz, Iran) at a controlled ambient temperature of $22 \pm 2^{\circ} \mathrm{C}$ with $50 \pm 10 \%$ relative humidity and a 12 -h light/12-h dark cycle. The animals were anesthetized by natrium pentobarbital $(50 \mathrm{mg} / \mathrm{kg}$; KELA Laboratoria NV, Hoogstraten, Belgium). The present study was performed in accordance with the Guide for the Care and Use of Laboratory Animals of Tabriz University of Medical Sciences, Tabriz, Iran (National Institutes of Health Publication No. 85-23, revised 1985).

Chemical reagents. Escherichia (serotype k235) lipopolysaccharide (LPS) and myeloperoxidase (MPO) were purchased from Sigma-Aldrich (St. Louis, MO, USA), and A-769662 from Tocris Bioscience (Bristol, UK). Rabbit monoclonal antibodies against phosphorylated (p)-AMPK $\alpha$ (Thr ${ }^{172}$; cat. no. $2535 ; 1: 1,000)$, AMPK $\alpha$ (cat. no. 5832; 1:1,000) and MyD88 (cat. no. 4283; 1:1,000) were obtained from Cell Signaling Technology, Inc. (Danvers, MA, USA). Mouse monoclonal GAPDH primary antibody (cat. no. mAbcam9484; 1:5,000), and peroxidase-conjugated goat anti-rabbit IgG - H\&L (HRP; cat. no. ab6721; 1:5,000) and rabbit anti-mouse IgG - H\&L (HRP; cat. no. ab6728; 1:5,000) secondary antibodies were obtained from Abcam (Cambridge, MA, USA), and Bender Med rat TNF- $\alpha$ ELISA from eBioscience, Inc. (San Diego, CA, USA). The protease inhibitor cocktail was purchased from Roche Diagnostics GmbH (Mannheim, Germany).

Experimental protocols. The rat model of LPS-induced inflammation was used as previously described (25) with minor modifications. The rats were divided into three groups $(\mathrm{n}=4)$ as follows: i) The normal control group, a vehicle-only, $80 \mu \mathrm{l}$ dimethyl sulfoxide (Merck Millipore, Darmstadt, Germany) to final volume of $1 \mathrm{ml}$ with normal saline; intraperitoneally injection (i.p.); ii) the LPS-treated group, LPS (0.5 mg/kg; i.p.); and iii) the LPS + A-769662-treated group, LPS (0.5 mg/kg; i.p.) and A-769662 (10 mg/kg; i.p.). The rats were weighed prior to treatment (time set at zero) and at the end of the experiment. At $9 \mathrm{~h}$ post LPS injection, the heart and lung tissues were removed. The harvested tissues were immediately rinsed in cold saline, snap-frozen in liquid nitrogen and stored at $-70^{\circ} \mathrm{C}$, or were directly fixed in formalin (Chem-Lab $\mathrm{NV}$, Zedelgem, Belgium) after rinsing for further analysis.

Measurements of TNF- $\alpha$ serum levels by ELISA. Serum levels of TNF- $\alpha$ were quantified using the ELISA kit according to the manufacturer's instructions. Briefly, blood was collected in a non-heparinized tube from the hepatic portal vein and serum was separated by centrifugation within 15 min of collection at $238.97 \mathrm{x} \mathrm{g}$ for $10 \mathrm{~min}$ at $15^{\circ} \mathrm{C}$. Serum was immediately aliquoted and stored at $-70^{\circ} \mathrm{C}$ until further analysis. The concentration of TNF- $\alpha$ serum levels are expressed as pg/ml of serum.

Neutrophil count. Prior to euthanasia, venous blood samples were collected to determine the number of neutrophils in the blood. A blood sample was smeared on a glass slide and the percentage of neutrophils was counted at a magnification of x100 using a CX31 optical microscope (Olympus Corporation, Tokyo, Japan) following Giemsa (Labtron Co., Tehran, Iran) staining. The percentage of neutrophils was calculated as a percentage of total white blood cells.

Measurement of MPO activity in heart and lung tissues. MPO activity was measured to quantify the activity of neutrophils in the tissues of interest as previously described (9), with minor modifications. Briefly, the tissues were sectioned in $50 \mathrm{mM}$ potassium phosphate buffer ( $\mathrm{pH}$ 6; Merck Millipore), containing $0.5 \%$ hexadecyl-trimethyl ammonium bromide (HTAB; Sigma-Aldrich) and homogenized for $3 \mathrm{~min}$ at 7,673.7 x g. The homogenates were sonicated using an ultrasonic cleaner (Starsonic 18-35, Bologna, Italy) for $10 \mathrm{sec}$, frozen and thawed 3 times, and then centrifuged at $2,150.7 \mathrm{x}$ g at $4^{\circ} \mathrm{C}$ for $45 \mathrm{~min}$. An aliquot of the supernatant $(0.1 \mathrm{ml})$ or standard 
was added to $2.9 \mathrm{ml}$ phosphate-buffered saline containing $0.167 \mathrm{mg} / \mathrm{ml}$ of O-dianisidine dihydrochloride and $0.0005 \%$ $\mathrm{H}_{2} \mathrm{O}_{2}$ (Merck Millipore). After $5 \mathrm{~min}$, the reaction was stopped with $0.1 \mathrm{ml} 1.2 \mathrm{M} \mathrm{HCl}$ (Merck Millipore) and absorbance was measured with a spectrophotometer (Cecil 9000, Cecil Instruments, Cambridge, UK) at $400 \mathrm{~nm}$. The concentrations were calculated using calibration curves and expressed as units of MPO in $100 \mathrm{mg}$ weight of wet tissue (mU/100 mg).

Lung histopathological examination. For the histopathological examination, samples of lung tissue were removed at the end of the experiment and fixed in 10\% neutral-buffered formalin. The tissues were embedded in paraffin, sectioned at $5 \mu \mathrm{m}$ and stained with hematoxylin and eosin (Labtron Co.) for assessment of tissue injury and neutrophil accumulation in the microvasculature of injured lungs.

Western blot analysis. Western blotting was performed as previously described (9), with minor modifications. Following the experimental procedure, myocardial and lung tissues were removed and immediately deep-frozen in liquid nitrogen. The tissue samples were homogenized in ice-cold solution ( $\mathrm{pH} 7.4$ ) containing $50 \mathrm{mM}$ Tris-HCl (Merck Millipore)., $150 \mathrm{mM}$ $\mathrm{NaCl}$ (Merck Millipore),, $5 \mathrm{mM}$ sodium pyrophosphate (Sigma-Aldrich), 50 mM NaF (Sigma-Aldrich), 1 mM EDTA (Merck Millipore)., $1 \mathrm{mM}$ dithiothreitol (Sigma-Aldrich), $0.1 \%$ sodium dodecyl sulfate (SDS; Merck Millipore) (w/v), 1\% TXT-100 (v/v; Sigma-Aldrich) and protease inhibitor cocktail. Lung tissue contains extracellular matrix that is resistant to homogenization. Thus, prior to tissue lysis, tissue was ground thoroughly with a pestle and mortar, in liquid nitrogen. Following homogenization in lysis buffer (Merck Millipore), to completely destruct the cell membrane, samples were sonicated 8-10 times, for 3-5 sec. Homogenized heart and lung samples were centrifuged at $10,621 \mathrm{x}$ g at $4^{\circ} \mathrm{C}$ for $10 \mathrm{~min}$ and $2,150.7 \mathrm{x} \mathrm{g}$ at $4^{\circ} \mathrm{C}$ for $45 \mathrm{~min}$, respectively. The supernatant was aliquoted and stored at $-70^{\circ} \mathrm{C}$ for further analysis. The Bradford Protein Assay kit (Sigma-Aldrich) was used to evaluate the protein concentrations in the supernatant. The samples were mixed with loading buffer [1 g SDS, 7 cc $1 \mathrm{M}$ Tris (pH 6.8), 3 cc glycerin and Bromophenol blue (all from Merck Millipore)] and subsequently boiled for $10 \mathrm{~min}$, at $100^{\circ} \mathrm{C}$. Protein samples $(50 \mu \mathrm{g})$ were loaded onto a SDS-polyacrylamide gel (Sigma-Aldrich) using a Min-Protean Tetra Cell system (Bio-Rad Laboratories, Inc., Hercules, CA, USA) to be separated by electrophoresis at $120 \mathrm{~mA}$. Separated proteins were transferred to an Immobilon-P membrane (EMD Millipore, Billerica, MA, USA) and blocked in 5\% non-fat milk in Tris-buffered saline with Tween-20 (all from Merck Millipore) at room temperature with gentle shaking, for $1 \mathrm{~h}$. The membranes were washed with the wash buffer [Tris base $(6.05 \mathrm{~g})+,\mathrm{NaCl}(8.76 \mathrm{~g})+$ Tween-20 $(1 \%)$ to $1 \mathrm{~L}$ by deionized water, $\mathrm{pH}$ 7.4] all from (Merck Millipore). The membranes were then incubated with the primary antibodies against p-AMPK $\alpha$ (Thr172), AMPK $\alpha, \operatorname{MyD} 88$ (1:1,000) and GAPDH $(1: 5,000)$ at $4^{\circ} \mathrm{C}$, with gentle shaking, overnight. The membranes were then washed and incubated with the peroxidase-conjugated goat anti-rabbit and rabbit anti-mouse secondary antibodies $(1: 5,000)$, at room temperature, with gentle shaking, for $1 \mathrm{~h}$. For phosphorylated proteins, blocking

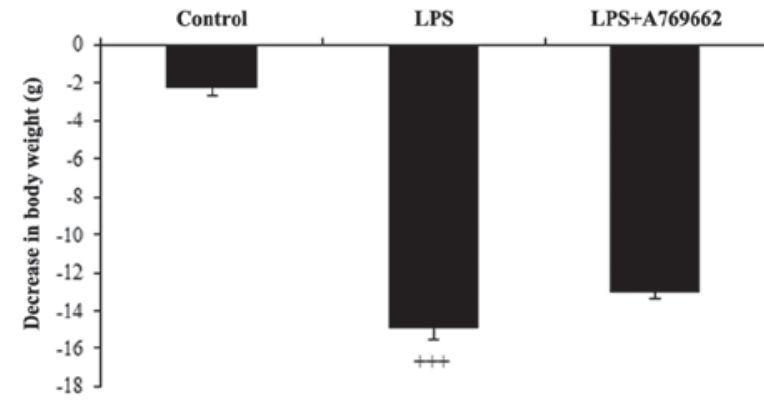

Figure 1. Effect of A-769662 treatment $(10 \mathrm{mg} / \mathrm{kg})$ on body weight reduction in control, LPS-treated and LPS + A-769662-treated rats. Data are presented as the mean \pm standard error $(n=4) .{ }^{++} \mathrm{P}<0.001$ vs. the control group. LPS lipopolysaccharide.

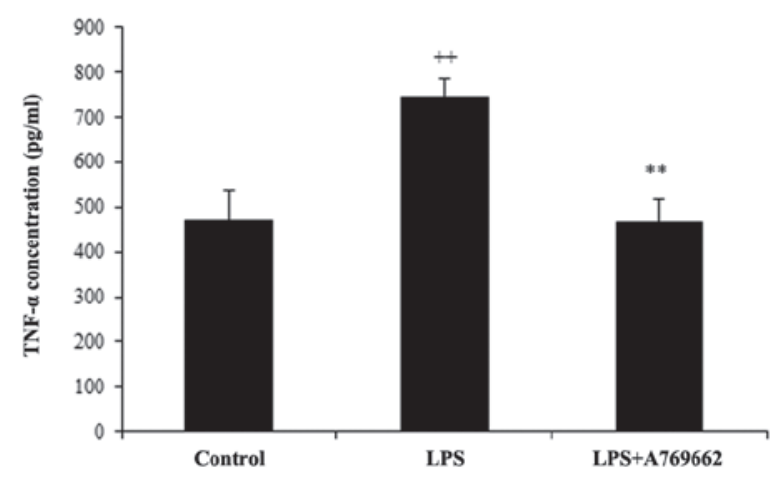

Figure 2. Effect of A-769662 treatment $(10 \mathrm{mg} / \mathrm{kg})$ on the concentration of TNF- $\alpha$ in the serum obtained from control, LPS-treated and LPS + A-769662-treated rats. Data are presented as the mean \pm standard error $(n=4) .{ }^{++} \mathrm{P}<0.01$ vs. the control group and ${ }^{* *} \mathrm{P}<0.01$ vs. the LPS-treated group. LPS, lipopolysaccharide; TNF- $\alpha$, tumor necrosis- $\alpha$.

buffer and antibodies diluents contained $50 \mathrm{mM} \mathrm{NaF}$ as anti-phosphatase. Subsequent to washing, antibodies were visualized using the BM Chemiluminescence Western Blotting kit (Roche Diagnostics $\mathrm{GmbH}$ ). Densitometric analysis of the immunoblots was performed using Image $\mathrm{J}$ software (version 1.41; National Institutes of Health, Bethesda, MD, USA). The densitometric values of $\mathrm{p}$-AMPK $\alpha$ were normalized to AMPK $\alpha$ and in the case of MyD88 to GAPDH.

Statistical analysis. Data are presented as the mean \pm standard error. One way analysis of variance (ANOVA) was used for comparison among the groups. If the ANOVA analysis indicated significant differences, the Fisher's least significant difference post-hoc test was performed to compare the mean values between the treatment groups and control. $\mathrm{P}<0.05$ was considered to indicate a statistically significant difference.

\section{Results}

Changes in body weight. Following administration of LPS, the rats demonstrated a general loss of appetite and reduction in water consumption. This resulted in a significant reduction in body weight of $14.9 \pm 1.6 \mathrm{~g}$, at $9 \mathrm{~h}$ subsequent to LPS injection, compared with the control group $(\mathrm{P}<0.001$; Fig. 1). As demonstrated in Fig. 1, compared with the LPS-only treated group, animals treated with A-769662 exhibited reduced weight loss. 


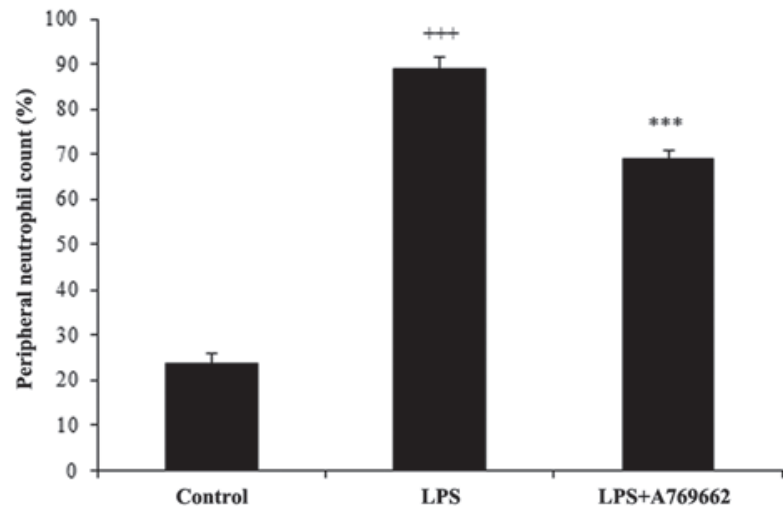

Figure 3. Effect of A-769662 treatment $(10 \mathrm{mg} / \mathrm{kg})$ on the neutrophil count in the blood obtained from control, LPS-treated and LPS + A-769662-treated rats. Data are presented as the mean \pm standard error $(\mathrm{n}=4) .{ }^{++} \mathrm{P}<0.001$ vs. the control group and ${ }^{* * *} \mathrm{P}<0.01$ vs. the LPS-treated group. LPS, lipopolysaccharide.

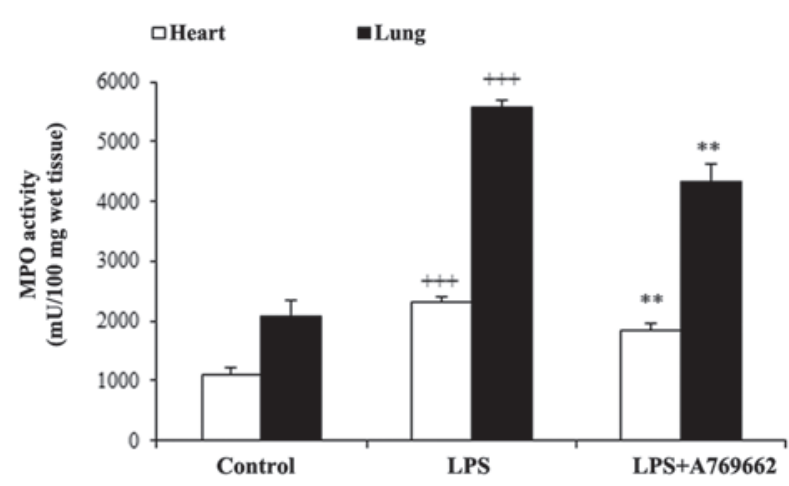

Figure 4. Effect of A-769662 treatment $(10 \mathrm{mg} / \mathrm{kg})$ on the myeloperoxidase activity in heart and lung tissues in control, LPS-treated and LPS + A-769662-treated rats. Data are presented as the mean \pm standard error $(n=4) .{ }^{+++} \mathrm{P}<0.001$ vs. the control group and ${ }^{* *} \mathrm{P}<0.01$ vs. the LPS-treated group. LPS, lipopolysaccharide; MPO, myeloperoxidase.

Effect of A-769662 on the serum levels of TNF- $\alpha$ following LPS injection. As demonstrated in Fig. 2, the serum levels of TNF- $\alpha$ were significantly increased from $468 \pm 69.4 \mathrm{pg} / \mathrm{ml}$ in the normal control group to $743 \pm 42.9 \mathrm{pg} / \mathrm{ml}$ in the LPS-treated group $(\mathrm{P}<0.01)$. The concentration of TNF- $\alpha$ in the serum of the LPS + A-769662 group was reduced to a level similar to that of the normal control group $(467.2 \pm 51 \mathrm{pg} / \mathrm{ml} ; \mathrm{P}<0.01$ compared with the LPS-only group).

Efect of A-769662 on the blood neutrophil count. Injection of LPS resulted in a prominent elevation in the percentage of neutrophils from $23.7 \pm 2.2 \%$ in the normal control group to $88.7 \pm 2.7 \%$ ( $\mathrm{P}<0.001$; Fig. 3). Administration of A-769662 significantly reduced the percentage of peripheral neutrophil to $68.9 \pm 2$ compared with the LPS-treated group $(\mathrm{P}<0.01$; Fig. 3).

Effect of A-769662 on the heart and lung MPO activity following LPS injection. A characteristic feature of acute endotoxemia is the accumulation of neutrophils in the target tissues, thus MPO activity was utilized as an index of neutrophil infiltration. As demonstrated in Fig. 4, MPO activity significantly increased
A

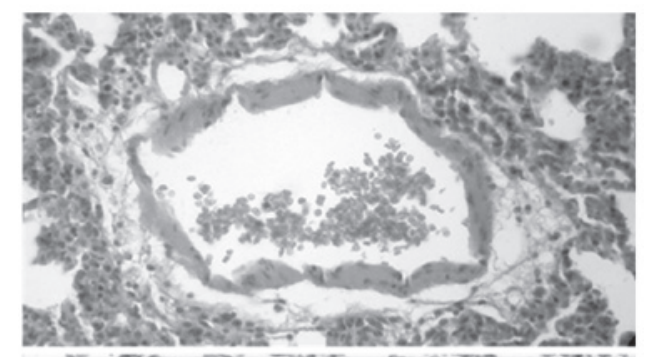

B

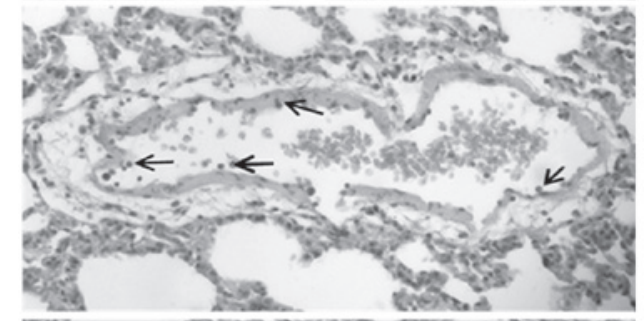

C

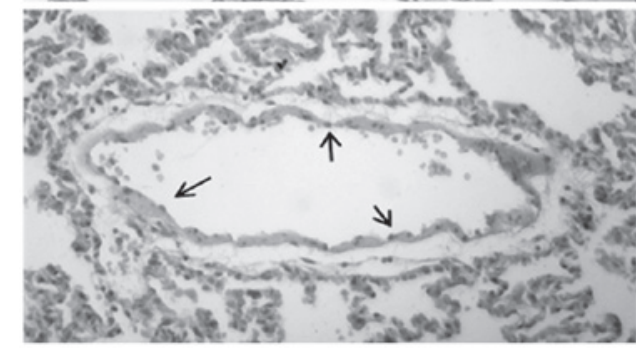

Figure 5. Neutrophil accumulation in the microvasculature of injured lungs following administration of A-769662 in LPS-induced rats. Sections were prepared from lung tissues excised $9 \mathrm{~h}$ after injection with (A) vehicle, (B) LPS $(0.5 \mathrm{mg} / \mathrm{kg})$ and (C) A-769662 (10 mg/kg) prior to LPS injection. The three captured images are each representative of three distinct experiments performed on 4 different rats. Arrows indicate neutrophils (hematoxylin and eosin, x40 magnification). LPS, lipopolysaccharide.

in heart and lung tissues in the LPS groups compared with the control groups $(\mathrm{P}<0.001)$. Additional treatment with A-769662 significantly reduced the MPO activity in the heart and lung tissues compared with the LPS-only group $(\mathrm{P}<0.01$; Fig. 4).

Histopathological examination of lung tissue. Microscopic examination of the endothelium of the lung tissue of LPS + A-769662-treated rats demonstrated reduced neutrophil accumulation compared with the LPS-only treated group (Fig. 5).

Effect of A-769662 on MyD88 protein expression levels in the heart and lung tissues of the rats injected with LPS. The protein expression levels of MyD88 were assessed to determine the effect of the treatments. As demonstrated in Fig. 6, $9 \mathrm{~h}$ subsequent to LPS injection, the protein levels of myocardial MyD88 were significantly increased compared with the control group $(\mathrm{P}<0.001)$. Additional treatment with A-769662 led to a significant reduction in the MyD88 protein expression levels compared with the LPS-only group ( $\mathrm{P}<0.01$; Fig. 6). Compared with the heart tissue, LPS was observed to have no effect on the content of MyD88 in the lung and there was no significant difference in the lung MyD88 levels between the LPS-only and LPS + A-769662 groups ( $>>0.05$; Fig. 7).

Effect of A-769662 on p-AMPKa protein expression levels in the heart and lung tissues of LPS-injected rats. AMPK is 

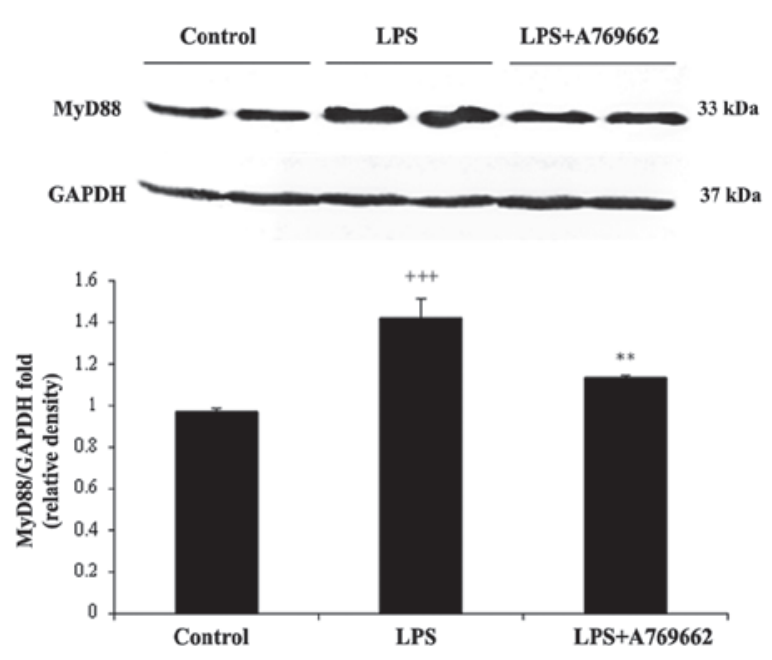

Figure 6. Representative immunoblots of MyD88 protein expression levels in the heart tissues of control, LPS-treated and LPS + A-769662-treated rats. Bars represent the ratio of MyD88 to GAPDH. Data are presented as the mean \pm standard error $(n=4) .{ }^{++} \mathrm{P}<0.001$ vs. the control group and ${ }^{* *} \mathrm{P}<0.01$ vs. the LPS-treated group. MyD88, myeloid differentiation factor 88; LPS, lipopolysaccharide; GAPDH, glyceraldehyde 3-phosphate dehydrogenase.

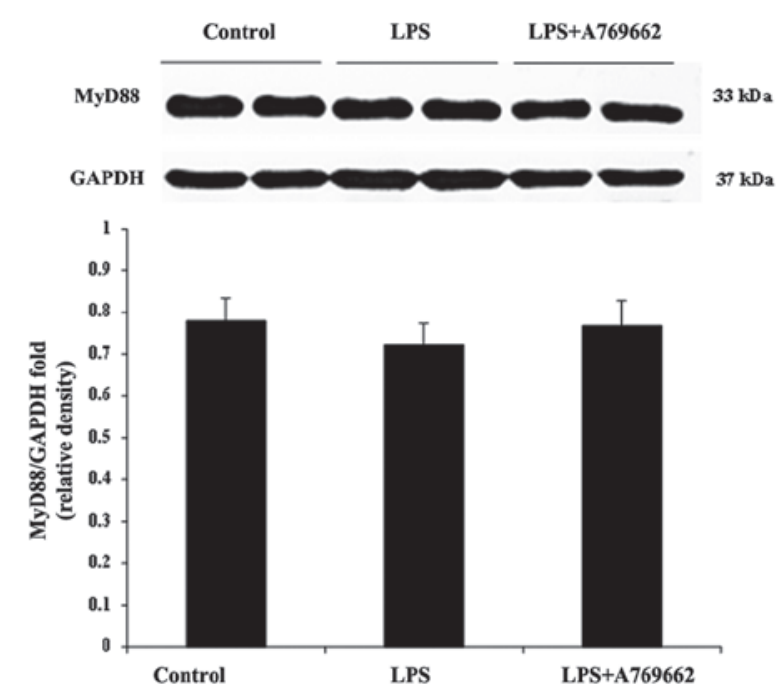

Figure 7. Representative immunoblots of MyD88 protein expression levels in the lung tissues of control, LPS-treated and LPS + A-769662-treated rats. Bars represent the ratio of MyD88 to GAPDH. Data are presented as the mean \pm standard error $(n=4)$. One way analysis of variance with Fisher's least significant difference post-hoc test demonstrated no significant differences between groups. MyD88, myeloid differentiation factor 88; LPS, lipopolysaccharide; GAPDH, glyceraldehyde 3-phosphate dehydrogenase.

an energy regulator present in various cells and its activation during metabolic stress, particularly inflammation, serves a role in cell survival. A-769662 is an established AMPK agonist, thus it was utilized for experimental purposes. As acute endotoxemia is associated with inflammation, the protein expression levels of $\mathrm{p}$-AMPK $\alpha$ were determined in the myocardial and lung tissues of LPS-injected rats with or without A-769662 treatment.

As demonstrated in Fig. 8, LPS treatment induced a notable AMPK activation in the heart tissue of rats. The relative expression of p-AMPK $\alpha$ to AMPK $\alpha$ in the LPS-treated group was significantly increased compared with that of the control
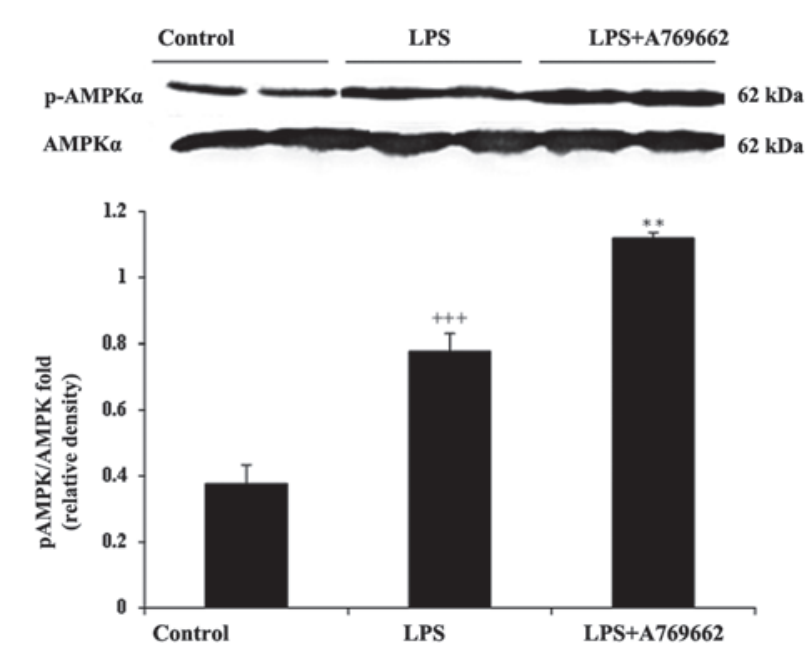

Figure 8. Representative immunoblots of $\mathrm{p}-\mathrm{AMPK} \alpha$ at residue threonine 172 and total AMPK $\alpha$ protein expression levels in the heart tissue of control, LPS-treated and LPS + A-769662-treated rats. Bars represent the ratio of phosphorylated AMPK $\alpha$ to total AMPK $\alpha$. Data are presented as the mean \pm standard error $(n=4) .{ }^{+++} \mathrm{P}<0.001$ vs. the control group and ${ }^{* *} \mathrm{P}<0.01$ vs. the LPS-treated group. p-AMPK $\alpha$, phosphorylated-AMP-activated protein kinase- $\alpha$; LPS, lipopolysaccharide.
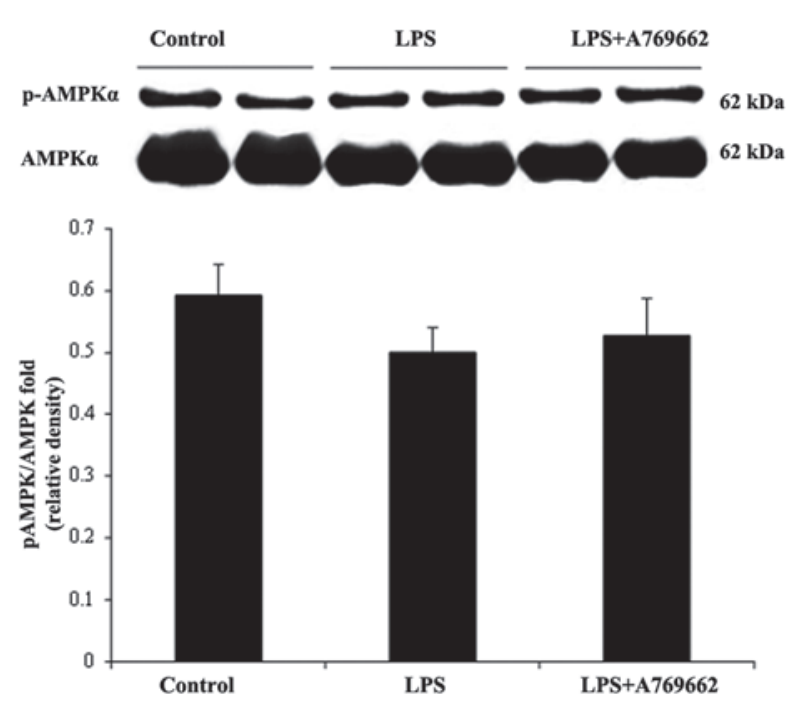

Figure 9. Representative immunoblots of p-AMPK $\alpha$ at residue threonine 172 and total AMPK $\alpha$ protein expression levels in the lung tissue of control, LPS-treated and LPS + A-769662-treated rats. Bars represent the ratio of phosphorylated AMPK $\alpha$ to total AMPK $\alpha$. Data are presented as the mean \pm standard error $(n=4)$. One way analysis of variance with Fisher's least significant difference post-hoc test demonstrated no significant differences between the groups. p-AMPK $\alpha$, phosphorylated-AMP-activated protein kinase- $\alpha$; LPS, lipopolysaccharide.

group ( $\mathrm{P}<0.001$; Fig. 8). Co-administration with A-769662 in the heart tissue of rats significantly enhanced the AMPK activation by LPS $(\mathrm{P}<0.01$; Fig. 8). However, as demonstrated in Fig. 9, no significant effect was observed in lung tissues following treatment with LPS or LPS + A-769662.

\section{Discussion}

The present study demonstrated that A-769662 inhibited the LPS-induced increase in the peripheral neutrophil count 
and MPO activity in the heart and lung tissues of the rats injected with the endotoxin. In addition, the administration of A-769662 significantly reduced the LPS-induced elevation of TNF- $\alpha$ concentration levels in the serum of the rats. Following LPS injection, levels of pro-inflammatory cytokines with a prominent role in endotoxin-induced organ injury (8), such as TNF- $\alpha$, rapidly increased in the blood $(25,26)$. For the results of the present study, MPO activity was used as an index of neutrophil infiltration and an increase in the activity levels was demonstrated in heart and lung tissues. Neutrophil accumulation in lung and heart tissues is a noticeable feature of acute endotoxemia (8). Excessive levels of LPS result in acute endotoxemia associated with the systemic inflammation and accumulation of macrophages in targeted tissues. Endotoxemia leads to septic shock, multiple organ damage and death (6-8), and pro-inflammatory cytokines, reactive oxygen and nitrogen species, proteases and bioactive lipids are considered as tissue damaging factors in endotoxemia (8).

AMPK serves a role in cellular energy homeostasis, and as a metabolic regulation enzyme, its activation during metabolic stress is important for cell survival (15). Previous studies have suggested that AMPK activation has a protective effect in inflammatory conditions (9,11-13). Furthermore, AICAR and metformin are indirect and nonspecific AMPK activators with a certain AMPK-independent effect (20), compared with A-769662 that is able to selectively and directly activate AMPK $(21,22)$ by binding to its $\beta$ subunit. This subunit is a site distinct from those of AMP, however, in a similar process to that of AMP, A-769662 allosterically activates AMPK and renders the phosphorylated Thr172 residue resistant to protein phosphatases (20).

AMPK activation has been demonstrated to be involved in the anti-inflammatory effect of the agonists in different models (11-13). In the current study, administration of A-769662 prior to LPS injection was demonstrated to suppress the neutrophil infiltration into the heart and lung tissues, and reduce the peripheral neutrophil count. A-769662 was demonstrated to activate AMPK through a mechanism involving the phosphorylation of a subunit of the enzyme (20), and previous studies indicated that the phosphorylation of AMPK may suppress the TLR4 expression and activity in conditions associated with inflammation, such as myocardial infarction $(9,10)$. In accordance with the activation of TLRs, in the present study the injection of rats with LPS led to a marked increase in the MyD88 protein expression levels in the heart tissue and a considerable elevation in the TNF- $\alpha$ serum levels. The LPS binding protein bound to endotoxins is recognized by the CD14/TLR4-MD-2 complex in the innate immune cells and delivers a signal through the plasma membrane (27). Stimulation of TLR4 facilitates the activation of MyD88, leading to nuclear translocation of $N F-\kappa B$ and the production of pro-inflammatory cytokines, including TNF- $\alpha$ and IL-6 (28). MyD88 is an adaptor molecule of the TLR4 pathway and a prominent part of the LPS receptor complex involved in the production of pro-inflammatory cytokines that lead to tissue injury. Furthermore, reactive oxygen and nitrogen species are destructive via products of endotoxemia (8), and their production through TLR4 and MyD88-dependent signaling may lead to oxidative stress via AMPK activation (15).
In support of these observations, the present study demonstrated that the increase of MyD88 protein expression and TNF- $\alpha$ serum levels in the heart tissue were significantly attenuated by A-769662 administration, suggesting suppressed TLR activity.

In addition to heart tissue, lung tissue is sensitive to LPS-induced endotoxemia (8). In the present study, LPS administration induced the elevation of MPO activity in the lung tissue. This effect was confirmed by lung histopathological analysis in which neutrophils sequestered onto the vessel firmly adhered to the endothelial wall. However, the levels of MyD88 and p-AMPK protein expression were not increased in the lung tissue following LPS administration. In accordance with these results and Lefort et al (29), LPS administration (i.p.) may trigger a signal at the systemic or heart level, but fails to induce a full signal to increase the levels of MyD88 or p-AMPK in the lungs. Additionally, the results of the current study demonstrated that the administration of A-769662 to the LPS-injected rats resulted in a significant reduction of MPO activity and neutrophil infiltration in the lung tissue, however, no effect was observed in the levels of MyD88 or p-AMPK.

To the best of our knowledge, this is the first study investigating the effect of A-769662 on the AMPK activity in the lung tissue. AMPK is a heterotrimer complex comprised of $\alpha$-, $\beta$ - and $\gamma$-subunits, each of which has two or more isoforms encoded by multiple genes and are differentially expressed in various types of tissue (17). The $\alpha 2$ and $\beta 2$ isoforms are highly expressed in the myocardium, and the $\alpha 1$ and $\beta 1$ isoforms are prominent in the lung (30). Additionally, the $\alpha 1, \beta 1$ and $\gamma 1$ isoforms are ubiquitously expressed. A-769662 selectively activates the AMPK heterotrimeric complex containing $\alpha 2 / \beta 1$ subunits (31) that may be noticeable in the myocardium and not in the lung tissue.

Previous studies demonstrated that activation of AMPK by metformin diminishes the cardiac inflammatory responses following myocardial infarction by suppressing the TLR4/MyD88 activity $(9,10)$. Salminen et al (32) demonstrated that the activation of AMPK inhibits NF- $\kappa \mathrm{B}$ activity, suppresses the expression of the pro-inflammatory cytokines and attenuates inflammatory injury through phosphorylation of downstream targets, including silent information regulator 1, PGC-1 $\alpha$, p53 and FoxOs. Furthermore, AMPK activation inhibits acute and chronic colitis (11), autoimmune encephalomyelitis (12), inflammation in cystic fibrosis (33), pro-inflammatory effects following lung injury (13) and LPS-induced expression of pro-inflammatory molecules and mediators (32). Stimulating autophagy (34) or inhibiting $\mathrm{NF}-\kappa \mathrm{B}$ activation (35) may be the mechanism underlying the regulation of inflammation by AMPK activation (32). The present study provided evidence that A-769662 reduces the systemic feature of LPS-induced endotoxemia.

In conclusion, the current study indicated that A-769662 protects against LPS-induced inflammatory responses in rats. The effect is associated with suppression of TLR activity in the heart tissue, potentially due to the increase in AMPK activity. Inhibition of neutrophil activity in the lung tissue was due to the inhibition of systemic inflammation by treatment with A-769662. The effect of A-769662 in the lung tissue was demonstrated to be independent of the AMPK activation and TLR suppression. Therefore, AMPK activation by A-769662 
and the reduction of systemic features of endotoxemia may be a promising target in the endotoxemia treatment.

\section{Acknowledgements}

The present study was supported by the Research Vice Chancellors of Tabriz University of Medical Sciences (Tabriz, Iran). The study was written based on the data of Maryam Rameshrad's Ph.D. thesis at Tabriz University of Medical Sciences (no. 88).

\section{References}

1. Akira S and Takeda K: Toll-like receptor signalling. Nat Rev Immunol 4: 499-511, 2004.

2. Takeda K: Evolution and integration of innate immune recognition systems: The Toll-like receptors. J Endotoxin Res 11: 51-55, 2005.

3. Beutler B: Inferences, questions and possibilities in Toll-like receptor signalling. Nature 430: 257-263, 2004

4. Cristofaro P and Opal SM: Role of Toll-like receptors in infection and immunity: Clinical implications. Drugs 66: 15-29, 2006.

5. Reitsma PH, Branger J, Van Den Blink B, Weijer S, Van Der Poll T and Meijers JC: Procoagulant protein levels are differentially increased during human endotoxemia. J Thromb Haemost 1 : 1019-1023, 2003

6. Ramana KV, Willis MS, White MD, Horton JW, DiMaio JM, Srivastava D, Bhatnagar A and Srivastava SK: Endotoxin-induced cardiomyopathy and systemic inflammation in mice is prevented by aldose reductase inhibition. Circulation 114: 1838-1846, 2006.

7. Jardin F, Brun-Ney D, Auvert B, Beauchet A and Bourdarias JP Sepsis-related cardiogenic shock. Crit Care Med 18: 1055-1060, 1990.

8. Connor AJ, Chen LC, Joseph LB, Laskin JD and Laskin DL: Distinct responses of lung and liver macrophages to acute endotoxemia: Role of toll-like receptor 4. Exp Mol Pathol 94: 216-227, 2013.

9. Soraya H, Farajnia S, Khani S, Rameshrad M, Khorrami A, Banani A, Maleki-Dizaji N and Garjani A: Short-term treatment with metformin suppresses toll like receptors (TLRs) activity in isoproterenol-induced myocardial infarction in rat: Are AMPK and TLRs connected? Int Immunopharmacol 14: 785-791, 2012.

10. Soraya H, Clanachan AS, Rameshrad M, Maleki-Dizaji N, Ghazi-Khansari $\mathrm{M}$ and Garjani A: Chronic treatment with metformin suppresses toll-like receptor 4 signaling and attenuates left ventricular dysfunction following myocardial infarction. Eur J Pharmacol 737: 77-84, 2014.

11. Bai A, Ma AG, Yong M, Weiss CR, Ma Y, Guan Q, Bernstein CN and Peng Z: AMPK agonist downregulates innate and adaptive immune responses in TNBS-induced murine acute and relapsing colitis. Biochem Pharmacol 80: 1708-1717, 2010.

12. Nath N, Giri S, Prasad R, Salem ML, Singh AK and Singh I: 5-aminoimidazole-4-carboxamide ribonucleoside: A novel immunomodulator with therapeutic efficacy in experimental autoimmune encephalomyelitis. J Immunol 175: 566-574, 2005.

13. Zhao X, Zmijewski JW, Lorne E, Liu G, Park YJ, Tsuruta Y and Abraham E: Activation of AMPK attenuates neutrophil proinflammatory activity and decreases the severity of acute lung injury. Am J Physiol Lung Cell Mol Physiol 295: L497-L504, 2008.

14. Shirwany NA and Zou MH: AMPK in cardiovascular health and disease. Acta Pharmacol Sin 31: 1075-1084, 2010.

15. Young LH, Li J, Baron SJ and Russell RR: AMP-activated protein kinase: A key stress signaling pathway in the heart. Trends Cardiovasc Med 15: 110-118, 2005.

16. Dyck JR and Lopaschuk GD: AMPK alterations in cardiac physiology and pathology: Enemy or ally? J Physiol 574: 95-112, 2006.

17. Hardie DG, Carling D and Gamblin SJ: AMP-activated protein kinase: Also regulated by ADP? Trends Biochem Sci 36: 470-477, 2011.
18. Hardie DG: Minireview: The AMP-activated protein kinase cascade: The key sensor of cellular energy status. Endocrinology 144: 5179-5183, 2003.

19. Kemp BE: Bateman domains and adenosine derivatives form a binding contract. J Clin Invest 113: 182-184, 2004.

20. Kim AS, Miller EJ, Wright TM, Li J, Qi D, Atsina K, Zaha V, Sakamoto K and Young LH: A small molecule AMPK activator protects the heart against ischemia-reperfusion injury. J Mol Cell Cardiol 51: 24-32, 2011

21. Cool B, Zinker B, Chiou W, Kifle L, Cao N, Perham M, Dickinson R, Adler A, Gagne G, Iyengar R, et al: Identification and characterization of a small molecule AMPK activator that treats key components of type 2 diabetes and the metabolic syndrome. Cell Metab 3: 403-416, 2006.

22. Göransson O, McBride A, Hawley SA, Ross FA, Shpiro N, Foretz M, Viollet B, Hardie DG and Sakamoto K: Mechanism of action of A-769662, a valuable tool for activation of AMP-activated protein kinase. J Biol Chem 282: 32549-32560, 2007.

23. Zhao X, Petursson F, Viollet B, Lotz M, Terkeltaub R and Liu-Bryan R: Peroxisome proliferator-activated receptor $\gamma$ coactivator $1 \alpha$ and FoxO3A mediate chondroprotection by AMP-activated protein kinase. Arthritis Rheumatol 66: 3073-3082, 2014

24. Petursson F, Husa M, June R, Lotz M, Terkeltaub R and Liu-Bryan R: Linked decreases in liver kinase B1 and AMP-activated protein kinase activity modulate matrix catabolic responses to biomechanical injury in chondrocytes. Arthritis Res Ther 15: R77, 2013.

25. Meng X, Ao L, Meldrum DR, Cain BS, Shames BD, Selzman CH, Banerjee A and Harken AH: TNF-alpha and myocardial depression in endotoxemic rats: Temporal discordance of an obligatory relationship. Am J Physiol 275: R502-R508, 1998.

26. Copeland S, Warren HS, Lowry SF, Calvano SE and Remick D; Inflammation and the Host Response to Injury Investigators: Acute inflammatory response to endotoxin in mice and humans. Clin Diagn Lab Immunol 12: 60-67, 2005.

27. Turyn D, Dominici FP, Sotelo AI and Bartke A: Specific interactions of growth hormone $(\mathrm{GH})$ with GH-receptors and GH-binding proteins in vivo in genetically GH-deficient Ames dwarf mice. Growth Horm IGF Res 8: 389-396, 1998.

28. Lu YC, Yeh WC and Ohashi PS: LPS/TLR4 signal transduction pathway. Cytokine 42: 145-151, 2008.

29. Lefort J, Singer M, Leduc D, Renesto P, Nahori MA, Huerre M, Créminon C, Chignard M and Vargaftig BB: Systemic administration of endotoxin induces bronchopulmonary hyperreactivity dissociated from TNF-alpha formation and neutrophil sequestration into the murine lungs. J Immunol 161: 474-480, 1998.

30. Kim M and Tian R: Targeting AMPK for cardiac protection: Opportunities and challenges. J Mol Cell Cardiol 51: 548-553, 2011.

31. Timmermans AD, Balteau M, Gélinas R, Renguet E, Ginion A, de Meester C, Sakamoto K, Balligand JL, Bontemps F, Vanoverschelde JL, et al: A-769662 potentiates the effect of other AMP-activated protein kinase activators on cardiac glucose uptake. Am J Physiol Heart Circ Physiol 306: H1619-H1630, 2014.

32. Salminen A, Hyttinen JM and Kaarniranta K: AMP-activated protein kinase inhibits NF- $\kappa \mathrm{B}$ signaling and inflammation: Impact on healthspan and lifespan. J Mol Med Berl 89: 667-676, 2011.

33. Myerburg MM, King JD Jr, Oyster NM, Fitch AC, Magill A, Baty CJ, Watkins SC, Kolls JK, Pilewski JM and Hallows KR: AMPK agonists ameliorate sodium and fluid transport and inflammation in cystic fibrosis airway epithelial cells. Am J Respir Cell Mol Biol 42: 676-684, 2010.

34. Egan DF, Shackelford DB, Mihaylova MM, Gelino S, Kohnz RA, Mair W, Vasquez DS, Joshi A, Gwinn DM, Taylor R, et al: Phosphorylation of ULK1 (hATG1) by AMP-activated protein kinase connects energy sensing to mitophagy. Science 331: 456-461, 2011

35. Hattori Y, Nakano Y, Hattori S, Tomizawa A, Inukai K and Kasai K: High molecular weight adiponectin activates AMPK and suppresses cytokine-induced NF-kappaB activation in vascular endothelial cells. FEBS Lett 582: 1719-1724, 2008. 